\title{
RECOGNIZING STATIC AND DYNAMIC SITTING BEHAVIOR BY MEANS OF INSTRUMENTED OFFICE CHAIRS
}

\author{
Bernhard Schwartz ${ }^{1}$, Andreas Schrempf ${ }^{1}$, Michael Haller $^{2}$, Kathrin Probst $^{2}$ and Josef Glöckl ${ }^{3}$ \\ ${ }^{1}$ Department of Medical Engineering, School of Applied Health and Social Sciences, \\ ${ }^{2}$ Media Interaction Lab, School of Informatics, Communications and Media, \\ University of Applied Sciences Upper Austria \\ ${ }^{3}$ aeris Impulsmöbel $\mathrm{GmbH} \& \mathrm{Co}$. KG \\ andreas.schrempf@fh-linz.at
}

\begin{abstract}
The increasing degree of computerization over the past years had led to higher amounts of sedentary time and time spent in static postures, which can be associated with higher risks of chronic diseases, obesity and even mortality. To counteract this negative developments a novel workplace conception called "Active Office" consisting of a standing and a sitting desk as well as an active chair is going to be developed. This new working place concept should motivate users to more physical activity by changing working posture and position whenever desired. An important part for the objective evaluation of this concept is the possibility to track the user's physical activity during several working days in comparison to a standard working place configuration. In this paper two prototypes of instrumented chairs are introduced, which allow to measure and quantify the sitting behavior of different persons. The devices consist of a smart sensor platform integrating sensors for posture measurement as well as the communication via a wireless protocol for data acquisition. The two prototypes were evaluated by means of a user study, where the sitting behavior of different users was recorded during several working days. It turned out that the instrumented office chairs are able to detect the user's physical activity within different working place configurations which provides a valuable basis for the further development and evaluation of the Active Office concept.
\end{abstract}

\section{KEY WORDS}

Activity Monitoring; Sedentary Behavior; Active Sitting; Sitting Posture

\section{Introduction}

\subsection{Motivation}

Regular exercise is a necessary part of physiological processes and therefore a crucial need for a healthy body. Physical diseases are often based on long periods of static sitting postures and a sedentary lifestyle. These factors often lead to diminished nutrient supply of the intervertebral discs, lower back pain (LBP), hypertension, diabetes, obesity [1, 2, 3] and therefore to a rising economic burden for the health-care system [4, 5, 6] up to an amount of 2.2 $\%$ of the gross domestic product [7]. To reduce this issues, novel workplace conceptions are focused on either different forms of standing desks or active chairs. Standing desks offer the possibility to work in natural posture with normal spine curvature and lower intervertebral disc loads [8] while active chairs provide the opportunity to work for a longer duration without getting physically fatigued. To benefit from both advantages an innovative workplace conception called "Active Office" is going to be developed. This concept combines standing and sitting desks with an active chair and therefore allows the user to change his working position whenever desired. This should motivate the user to increase his physical activity during a working day allowing a barrier-free movement between different working positions (sitting and standing). Moreover, this even meets the requirements for novel working places as stated by several health insurance companies [9].

\subsection{Lower Back Pain and Sedentary Behavior}

Lower back pain (LBP) is one of the most frequent diseases in today's society. In the past the main reason for LBP was a high vertebrae stress caused by lifting heavy loads in poor postures. Nowadays, more and more people - especially office workers - suffer from LBP caused by long occupational sedentary time and static sitting postures. This development becomes obvious when observing the average occupational activity profile of a common adult in the past. The Metabolic Equivalent of Task (MET) is one common possibility to quantify physical activity where $1 M E T=1 \mathrm{kcal} / \mathrm{kg} / \mathrm{h}$ which was intensively used by Ainsworth [10].The common weekly physical activity in the United States of America fell from about 150 METhours/week in 1965 to approximately 90 MET-hours/week in 2009. At the same time the common sedentary time rose from $26 \mathrm{~h} /$ week (1965) to $37 \mathrm{~h} /$ week (2009) [3]. Similar tendencies can be observed in other industrial countries. To counteract this negative development it is necessary to reduce sedentary time and simultaneously improve the sitting posture during the remaining sedentary time.

A very common way to reduce occupational sedentary time is a partial usage of standing desks. Current 
problems of such concepts are poor usability and bad performance due to considerable barriers when switching between workstations [11]. Furniture like active chairs that support micro-movements seem to be an essential part for improving sedentary behavior. It has been shown that active sitting can positively influence the prevalence of chronic spine curvatures [12] and improve sitting posture [13]. In order to assess the user's sitting behavior, thereby enabling the user to get adequate feedback, two instrumented office chairs were developed. This makes it possible to monitor the sitting behavior on a standard office chair as well as on an active chair.

The aim of this paper is to evaluate the developed instrumented office chairs as well as to investigate the sitting behavior of different persons by means of a user study. The work presented herein is part of a broader research activity with the aim to develop and evaluate a novel office environment called "Active Office". This novel office environment is going to combine a standing desk, a sitting desk with an active chair as well as different human-computer-interface (HCI) concepts [14], [15] and hence provides opportunities for office workers to seamlessly change between different work environments. As a result the "Active Office" is going to support people to carry out physically active work processes in a more natural way which in turn allows to prevent health problems associated with workplace induced inactivity [2]. In order to evaluate the "Active Office" against standard office environments it is essential to be able to assess the person's sitting behavior.

The paper is organized as follows: Section 2 focuses on the sensor concept, developed for two chairs (a standard office chair and an active chair respectively) in order to monitor the person's sitting position. Then a penaltyfunction approach for classifying the sitting posture with respect to position and activity is introduced and validated by means of laboratory experiments. Finally the key data of a user study for investigating the sitting behavior of different persons is provided. Section 3 provides the results of the incorporated user study focusing on static and dynamic sitting behavior and the ability of the penalty-function approach to classify the sitting behavior. Finally section 4 draws the main conclusions as well as provides an outlook to future research activities.

\section{Materials and Methods}

\subsection{Prototype of an Active Office}

The used workplace concept is a prototype of the "Active Office" (see Figure 11. It contains two height-adjustable desks, one whiteboard and one active chair called "Swopper" developed by Aeris (aeris - Impulsmöbel GmbH \& Co. KG, Haar, Germany). Each desk is equipped with a monitor, a keyboard and a mouse. To create different workplaces each one is adjusted to different heights depending on the standing and sitting elbow height as suggested by ergonomists. To increase storage space both desks are equipped with vertical shelves. Depending on the user's habits both monitors are operating in "extended" or "cloned" mode.

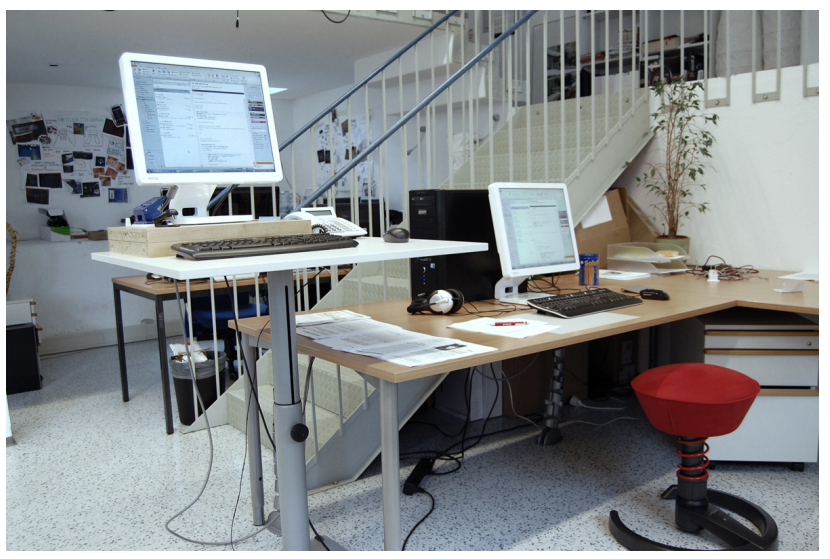

Figure 1. Prototype of an Active Office consisting of a standing and sitting desk as well of an active chair "Swopper" (aeris - Impulsmöbel GmbH \& Co. KG, Haar, Germany).

\subsection{The instrumented chairs}

In contrast to [16] or [17]we aim to develop a simple, affordable approach which allows to use conventional office chairs. The setup for the common office chair (see [18]) is based on a regular adjustable office chair, equipped with four independent, especially designed force transducers. The signal of the force transducers is digitized by a time-to-digital converter (ACAM Messtechnik $\mathrm{GmbH}$ ) and is further processed and sent by a micro-controller (NEON, spantec $\mathrm{GmbH}$ ) via the wireless ANT ${ }^{\mathrm{TM}}$ protocol. The four force transducers are located at each corner under the seating support, thus making it possible to compute the coordinates of the Center of Pressure $(C O P)$ with the reference frame located in the center of the seating support. The positive $x$-axis points towards the right side of a subject sitting on the chair and the $y$-axis faces towards the toes. The po-
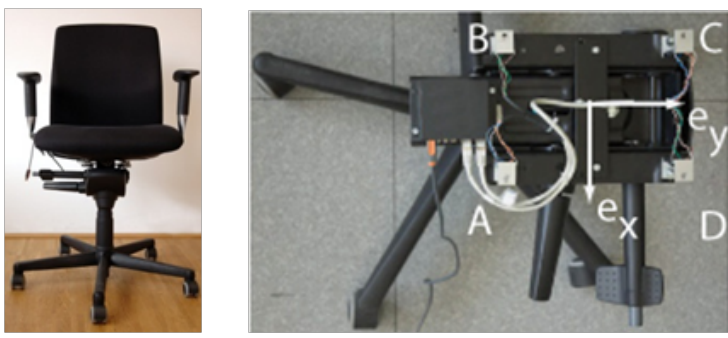

Figure 2. (left) The equipped office chair (right) Support frame of the chair. The 4 sensors are marked with the letters A-D, the reference frame is located in the center and labeled with $e_{x}$ and $e_{y}$ for the corresponding positive direction. 
sition of the COP corresponding to the reference frame in Figure 2 can be determined by

$$
\vec{r}_{C O P}=\left(\begin{array}{c}
C O P_{x} \\
C O P_{y}
\end{array}\right)=\frac{1}{F_{S}} \sum_{i=1}^{4} \vec{r}_{i} F_{i}
$$

where $F_{i}$ denotes the force measured by the $i$-th force transducer at position $\vec{r}_{i}=\left(r_{i, x} r_{i, y}\right)^{T}$. The resulting force is given by $F_{S}=\sum_{i=1}^{4} F_{i}$. The coordinates of the COP vary according to the person's posture on the chair which in turn allows to classify the sitting posture and the time spent in the corresponding position. For further analysis the mean values of the positions are computed and normalized by the range of motion (ROM). Additionally the normalized deviations of the positions from the mean values are computed and provided for further analysis as described below.

The setup for the active office chair called "Swopper" uses a tri-axial acceleration sensor for the detection of movements in three dimensions and exhibits a conical ROM (Figure 3). The acceleration signal is digitized and further processed by micro-controller mentioned above. Typically the sensor is mounted under the seating surface.

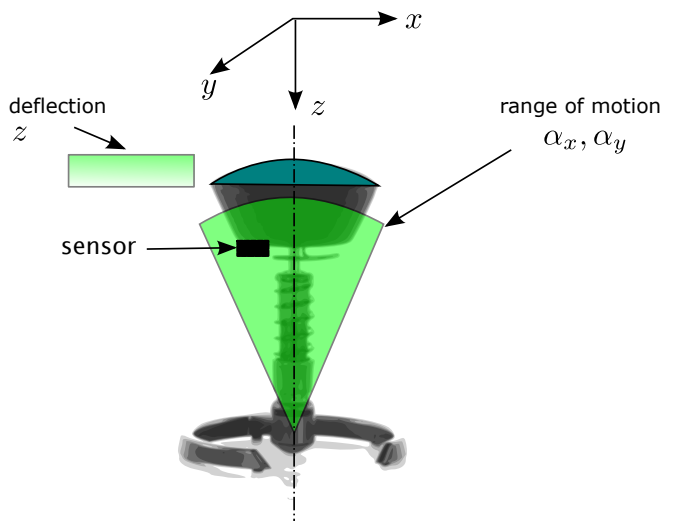

Figure 3. Reference frame, sensor position and range of motion of an active chair called "Swopper" .

To avoid measurement errors induced by variations in sensor positions an initial routine after activating the system provides the calibration of the system. By means of a lowpass filter with a cut-off frequency of $0.5 \mathrm{~Hz}$ first the mean accelerations are computed. Subsequently, the Euler angles are calculated and the filtered accelerations are transformed into the global reference system of the chair.

In operation mode the mean values of the angles $\alpha_{x}$ and $\alpha_{y}$ are computed by means of the raw acceleration measurements. First the acceleration values are averaged by means of a low-pass filter with a cut-off frequency of 0.5 Hz. As movements in the sagittal and frontal plane alter accelerations along circular orbits, the resulting angles can be calculated by use of common trigonometric functions (see Figure 4, left path). In order to obtain standardized results, the angles are finally normalized by the ROM.
The deviations from these mean values are given by $\Delta \alpha_{x}, \Delta \alpha_{y}$ and $\Delta z$ respectively. After rotating the acceleration values to the global reference frame, the deviations are obtained by means of a high-pass filter with a cut-off frequency of $0.5 \mathrm{~Hz}$. The acceleration values are transformed into angles and are then normalized by the ROM. For obtaining $\Delta z$ the vertical, transformed acceleration is integrated two times in order to obtain the vertical deflection. To avoid offset drifts due to bias, prior to each integration step an appropriate high-pass filter is applied (see Figure 4. right path). The mean angles $\alpha_{x}$ and $\alpha_{y}$ as well as
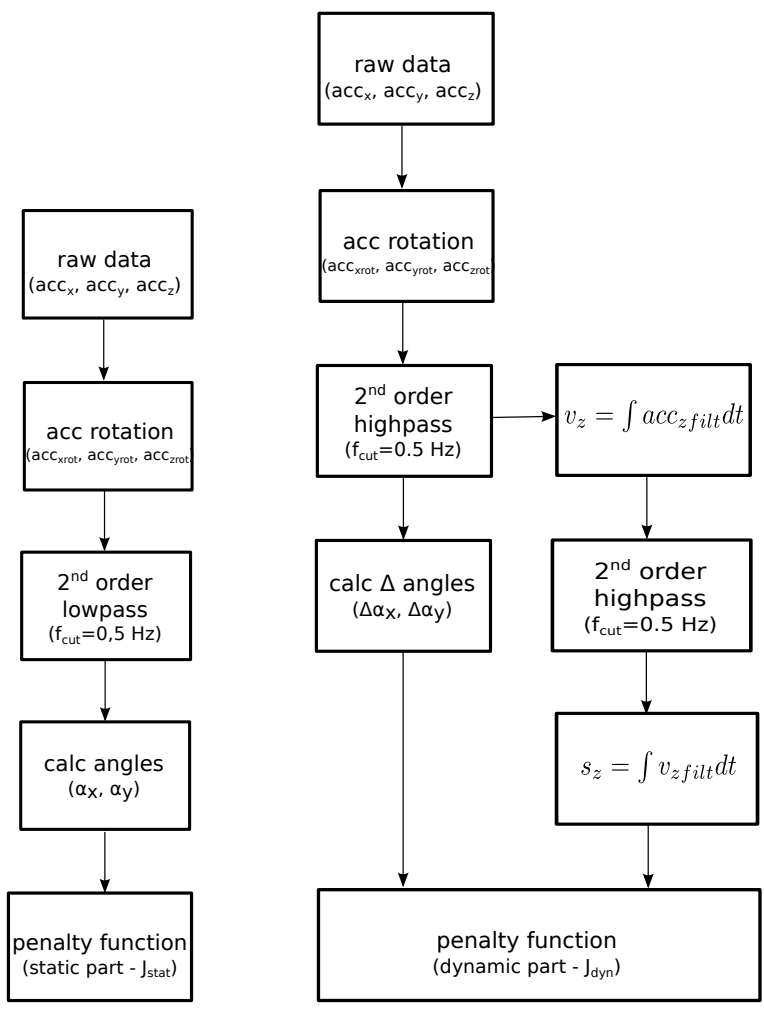

Figure 4. (left) mean values and static part $J_{\text {stat }}$ of cost function, (right) deviations and dynamic $J_{d y n}$ part of the cost function.

the deviations $\Delta \alpha_{x}, \Delta \alpha_{y}$ and $\Delta z$ respectively build the basis for posture classification by means of a penalty-function approach [18] .

\subsection{Posture Classification}

In contrast to [19] or [17] our goal is not to exactly classify the sitting postures, since a reliable classification requires additional sensors which are for example able to detect the curvature of the spine. Our goal was to develop an affordable sensor concept which can be simply applied to any available office chair. Since for the common static office chair as well as for the active chair the mean, normalized position values and its deviations are available, a penalty function $J$ can be computed for both setups, which consists of a static $J_{\text {stat }}$ and a dynamic part $J_{d y n}$ (see Figure 

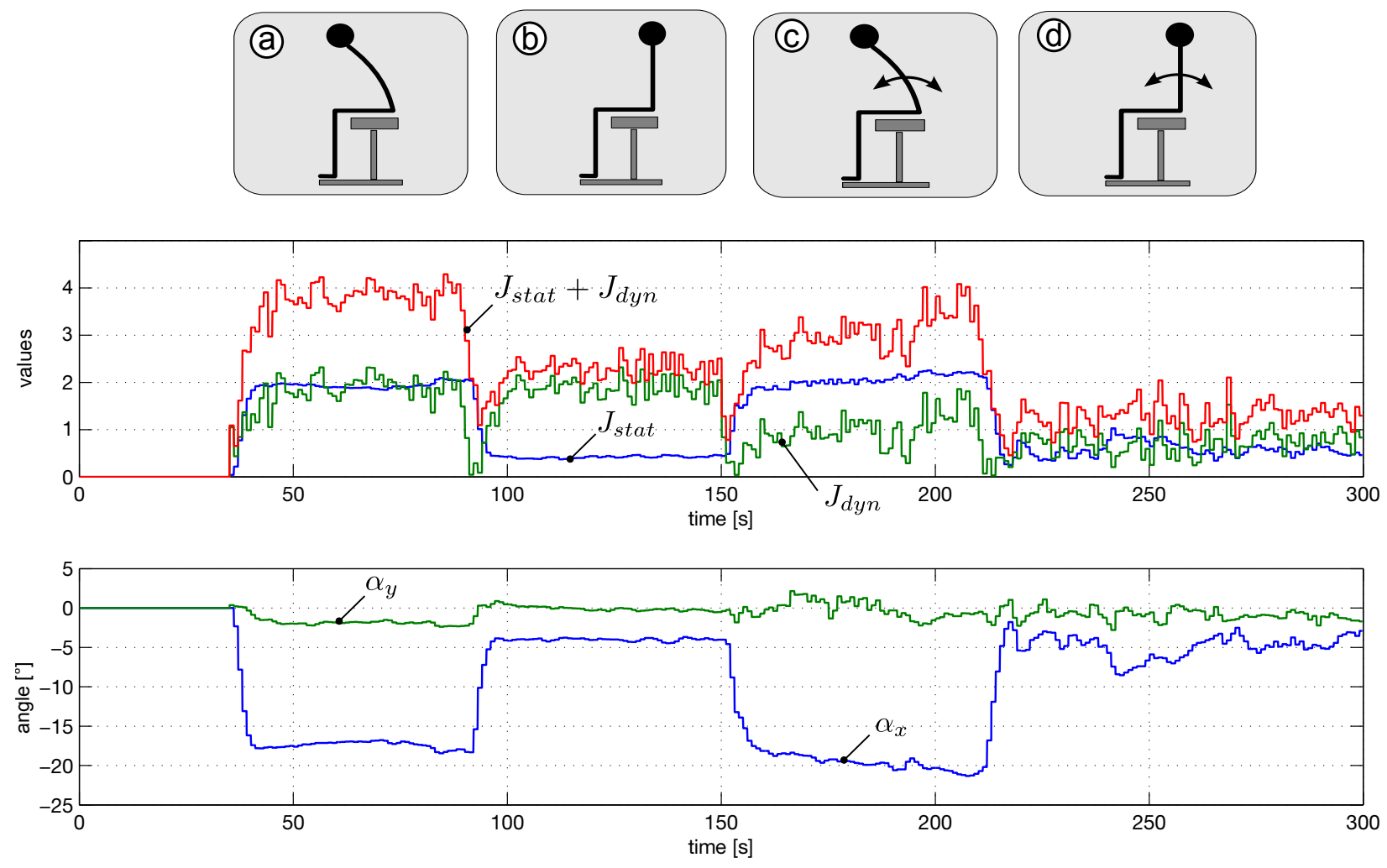

Figure 5. Time plot of penalty function $J$ (top) and chair angles (bottom) for the active chair for various sitting postures. Notice that the angles $\alpha_{x}$ and $\alpha_{y}$ are mean angles with respect to the global reference frame obtained by filtering.

4) defined by

$$
\begin{aligned}
J_{\text {stat }}= & C_{1} \sum_{i \in\{x, y\}}\left\|\alpha_{i}\right\|_{1} \\
J_{d y n}= & C_{21} \sum_{i \in\{x, y\}} \exp \left[-\left(C_{3 i}\left\|\Delta \alpha_{i}\right\|_{1}\right)\right]+ \\
& C_{22} \exp \left[-\left(C_{33}\|\Delta z\|_{1}\right)\right] .
\end{aligned}
$$

Hereby $C_{i}$ and $C_{i j}$ denotes weighting parameters which have to be determined arbitrarily. The $1-$ norm of $N$ measurements of the variable $x$ is defined by $\|x\|_{1}:=$ $\sum_{i=1}^{N}|x(i)|$. The higher the corresponding parts of the penalty function $J$ the more "unhealthy" the sitting posture is considered to be. The static part $J_{\text {stat }}$ provides a sensible parameter, which reflects the load acting on the spine. The load acting on the spine segments is basically reflected by the flexion/extension angles. With an increasing flexion angle the tendons around the spine are stretched and as a consequence the load on the spine segments increases [20]. Furthermore, as the flexion increases the force acting on the spine grows due to a larger moment arm of the gravity forces. Additionally, the vertebral discs are anterior more and more deformed with an increasing flexion angle, which in turn results in a higher load on the internal structure of the disc, especially on the annulus fibrosus which consists of several layers of collagen fibers in order to provide stability against bulging.
Very important is the fact that during a prolonged static sitting period the metabolism of the vertebral discs cannot work as required, since the transport of nutrients to the nucleus pulposus as well as the transport of waste out of the disc requires a periodic loading and releasing of the disc. Prolonged static sitting periods lead to a strongly reduced metabolism which in turn weakens the internal structure of the vertebral disc and makes it prone to failure [21]. Hence the deviation of the positions from their mean values forms another parameter proposed for posture classification which is considered by the dynamic part $J_{d y n}$.

The different measurement variables $C O P_{x}$, $C O P_{y}$ and $F_{S}$ with the static office chair, $\alpha_{x}, \alpha_{y}$ and $z$ with the active chair as well as the dynamic and static part of the penalty function $J_{\text {stat }}$ and $J_{d y n}$ respectively are computed in real-time on the micro-controller. Then they are sent wirelessly via the ANT-protocoll to any desktop computer or laptop equipped with an ANT USB-stick, which receives the data packets. A data server application based on a client/server architecture provides a real-time data stream via TCP/IP for further data processing with e.g. MatLab ${ }^{\mathrm{TM}}$. Since the data stream is provided via TCP/IP the data server and the client can be on different computers and at different locations. In MatLab ${ }^{\mathrm{TM}}$ a socket-connection to any (remote) TCP/IP data server 
based on Java provides the raw data for further algorithm development as well as for measurement studies.

\subsection{Laboratory Experiments}

To determine the weighting parameters of the penalty functions first measurements under controlled conditions were carried out. After an initialization the subjects remained in four predefined postures.

a) Sitting statically at the edge of the sitting support,

b) Sitting statically in the center of the sitting support,

c) Sitting dynamically at the edge of the sitting support,

d) Sitting dynamically in the center of the sitting support.

Figure 5 illustrates the time plots of the penalty function $J_{\text {stat }}$ and $J_{d y n}$ as well as the angles $\alpha_{x}$ and $\alpha_{y}$ while moving according to the previously defined protocol. Note that the angles $\alpha_{x}$ and $\alpha_{y}$ are mean values of the positions and reflect the mean value of the position in a predefined time frame. When sitting near the center of the chair $(b, d)$, the static penalty function $J_{\text {stat }}$ exhibits very low values. A very flexed posture or extended sitting posture is penalized by higher values of $J_{\text {stat }}$. Once reaching the borders of the ROM (angles about $20^{\circ}$ ) the values rise up to a maximum value of 2 . Active sitting is recognized by the dynamic part of the penalty function $J_{d y n}(\mathrm{c}, \mathrm{d})$ where the smallest values can be achieved. A very static sitting behavior $(a, b)$ is once again penalized by higher values of $J_{d y n}$.

\subsection{User study}

In a pilot project a user study with $N=12$ participants ( 9 female and 3 male) was carried out. Mean $( \pm S D)$ age was $32.75( \pm 9.25)$ years, height $172.33( \pm 7.23) \mathrm{cm}$, weight $71.96( \pm 10.84) \mathrm{kg}$ and BMI $24.17( \pm 2.77) \frac{\mathrm{kg}}{\mathrm{m}^{2}}$. Each participant was informed about the study, signed a consent form, obtained an instrumented chair ( 4 active chairs and 8 common office chairs) and was asked to use the system during normal office hours. For the user study we stated two goals a) to investigate with which feedback modality a user should be interrupted in case of an unhealthy sitting condition, and b) to learn about the sitting behavior of different participants on different chairs (common office chair vs. active chair). The analysis of three different feedback modalities i) graphical feedback, ii) physical feedback by means of an physical avatar as well as iii) vibro-tactile feedback is provided in [14] and is beyond the scope of this paper. Herein we will concentrate on the analysis of the different sitting behavior with respect to static and dynamic sitting depending on day-time and office chair.

For each participant the position was measured by means of two developed instrumented chairs, where the mean values and the deviations from it as well as the penalty functions $J_{\text {stat }}$ and $J_{d y n}$ according to eq. (2) and (3) were computed within subsequent time-windows of 1 minute. Hence for a normal working day of 8 hours we obtained 480 different samples containing the sitting behavior of one participant. The user study was divided in two parts, where in the first 2 weeks the users were allowed to become familiar with the system and the logging software, followed by $4-6$ weeks of data acquisition.

\section{Results and Discussion}

From the obtained data records within the user study the two parts $J_{\text {stat }}$ and $J_{d y n}$ of the penalty function were classified into three different regions.

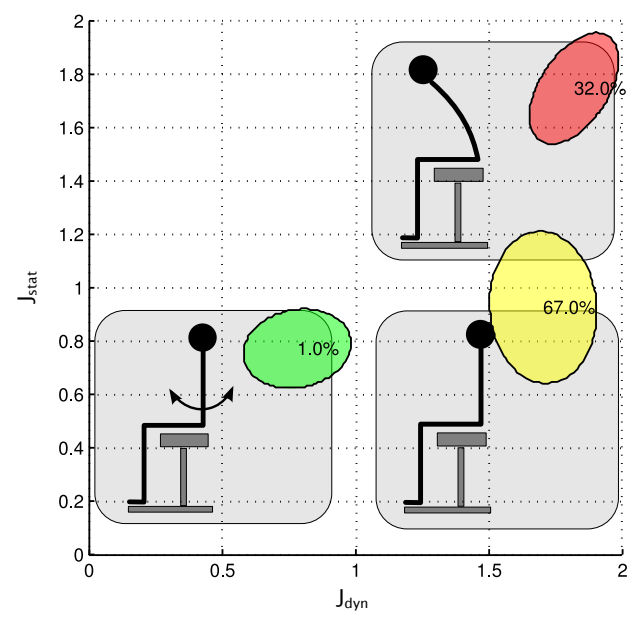

Figure 6. Distribution in percent during 4 weeks into three different regions - participant \#10 with a common office chair.

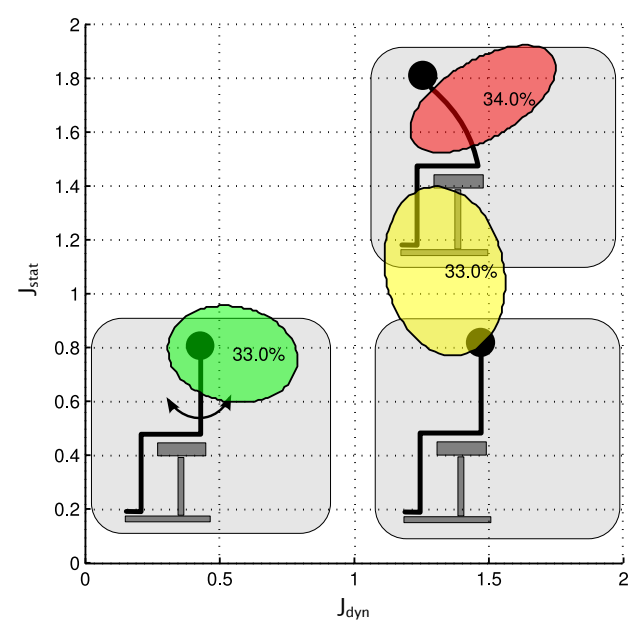

Figure 7. Distribution in percent during 4 weeks into three different regions - participant \#5 with an active chair.

Region 1 is defined by $0 \leq J_{\text {stat }} \leq J_{\text {stat,thres }}$ and $0 \leq J_{d y n} \leq J_{\text {dyn,thres }}$ which represents a dynamic sitting behavior. Region 2 is defined by $J_{\text {stat }}>J_{\text {stat,thres }}$ 
and $0 \leq J_{d y n} \leq J_{d y n, \text { thres }}$ which represents static sitting in a neutral position. Finally, region 3 defined by $J_{\text {stat }}>J_{\text {stat,thres }}$ and $J_{d y n}>J_{\text {dyn,thres }}$ represents static sitting in a flexed or extended position which is considered as the most unhealthy sitting configuration. The covariance matrix $P$ of the classified data samples is represented by an ellipsis with axes-directions defined by the eigenvectors of $P$ where the size of the major and minor axis is provided by the inverse of its eigenvalues. Figure 6 and Figure 7 illustrate the distribution of sitting in the three different regions for a participant using a common static office chair and an active chair respectively over a period of 4 weeks. It can be observed that participant \#10 with a static office chair achieves only $1 \%$ of dynamic sitting (region 1 ), sits about $67 \%$ in a neutral, but static position (region 2) and $32 \%$ in a static and a flexed position (region 3). A further analysis shows, that most of the time participant \#10 sits on the edge of the sitting support and rarely changes his position.

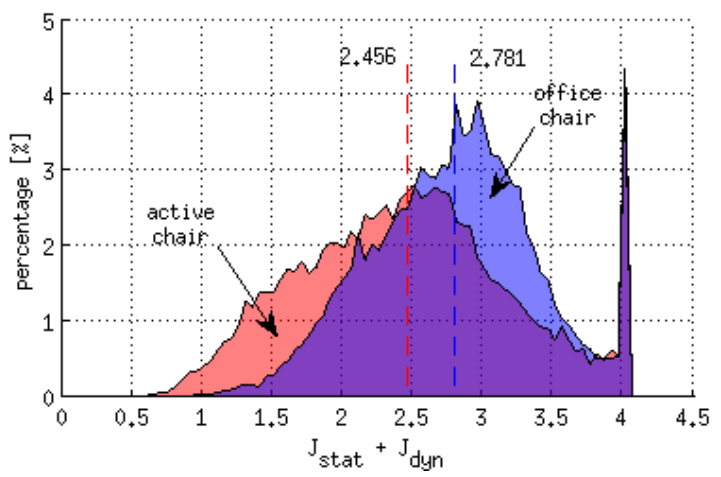

Figure 8. Comparison of the two chairs - histogram of $J=$ $J_{\text {stat }}+J_{d y n}$.
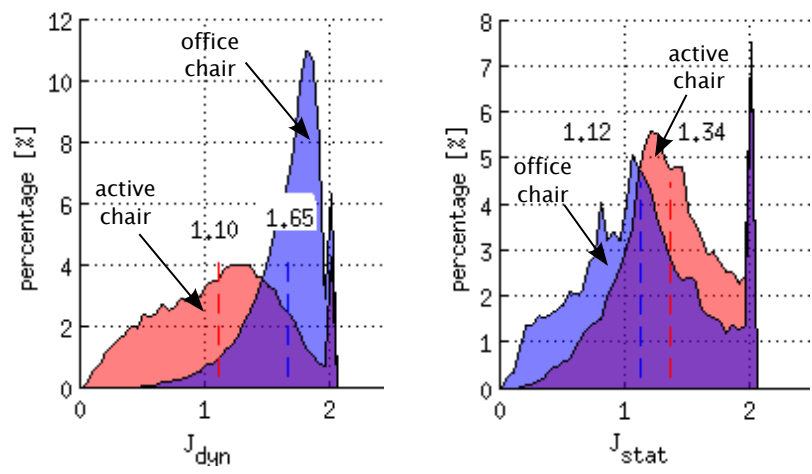

Figure 9. Comparison of the two chairs - histogram of $J_{d y n}$ and $J_{\text {stat }}$ respectively.

On the contrary participant \#5 who uses an active chair achieves $33 \%$ of dynamic sitting, $33 \%$ of sitting in a neutral, but static position and sits $34 \%$ in a static, flexed position. Most of the time participant \#5 sits near the cen- tral position with a 5 times higher range of motion than participant \#10.

Figure 8 shows the histograms of the cumulative penalty function $J=J_{\text {stat }}+J_{d y n}$ and Figure 9 depicts the penalty functions $J_{d y n}$ and $J_{\text {stat }}$ respectively summarized for all participants of the user study. It can be observed that participants using the active chair achieve a significantly smaller mean $\bar{J}=2.45$ of the cumulative penalty function than participants using the standard office chair with $\bar{J}=2.78$. When analyzing only the dynamic part $J_{d y n}$ of the penalty function, where participants sitting on the active chair achieve $\bar{J}_{d y n}=1.10$ compared to participants sitting on the common office chair $\bar{J}_{d y n}=1.65$, the hypothesis that a well-designed active chair helps the user to sit in a more active way, is mainly supported. On the other hand, Figure 9 shows that even with an active chair it is possible to sit statically, as the peak at $J=4$ indicates, which appears for both chairs. In that case the participants were sitting statically at the edge of the sitting support, which in certain cases lead to a saturation of the measurement system. Penalty function $J_{\text {stat }}$ in Figure 9, which reflects the mean position of the participants, indicates that participants with an active chair tend to lean slightly forward. But in contrast to a common office chair with fixed siting support, this does not lead to a flexed posture of the spine. By means of a spring strut the active chair allows a movement in all three dimensions, where even with an inclined position the spine remains at its natural curvature.

\section{Conclusions and Outlook}

In this paper two prototypes of instrumented chairs were introduced. They allow to measure and quantify the sitting behavior of different users. The posture classification was realized by incorporating a penalty function approach which penalizes static and flexed sitting on the one hand and rewards dynamic sitting in a central position. A user study with 12 participants proved the applicability of the developed instrumented chairs and allowed to investigate the different sitting behavior of the participants on different chairs. It turned out that the active chair promotes dynamical sitting which is reflected by a significantly lower mean value of the cumulative penalty function. On the other hand we can conclude that even with an active chair it is possible to sit statically.

Based on these major observations future work will extend the investigation of sitting behavior to the Active Office setting that includes a standing desk as well. Since the proposed instrumented chairs are only able to detect the sitting behavior, an extension of the sensor concept to the standing desk is important. This can be achieved by portable acceleration sensors [22], which allow to measure the participant's movements from sitting to standing and vice versa. 


\section{Acknowledgments}

The authors greatly acknowledge the financial support of the Austrian Research Promotion Agency - FFG. This publication is part of the FFG Bridge project "Active Office", project number 834185 .

\section{References}

[1] Sarah Kozey-Keadle, Amanda Libertine, John Staudenmayer, and Patty Freedson. The Feasibility of Reducing and Measuring Sedentary Time among Overweight, Non-Exercising Office Workers. Journal of obesity, 2012:282303, January 2012.

[2] N Owen, A Bauman, and W Brown. Too much sitting: a novel and important predictor of chronic disease risk? British journal of sports medicine, 43(2):8183, February 2009.

[3] K I Proper, E Cerin, W J Brown, and N Owen. Sitting time and socio-economic differences in overweight and obesity. International journal of obesity (2005), 31(1):169-76, January 2007.

[4] Simon Dagenais, Jaime Caro, and Scott Haldeman. A systematic review of low back pain cost of illness studies in the United States and internationally. The spine journal : official journal of the North American Spine Society, 8(1):8-20, 2008.

[5] Thomas Läubli and Christian Müller. Arbeitsbedingungen und Erkrankungen des Bewegungsapparates - geschätzte Fallzahlen und Kosten für die Schweiz. Die Volkswirtschaft Das Magazin für Wirtschaftspolitik, 11:22-25, 2009.

[6] N Maniadakis and a Gray. The economic burden of back pain in the UK. Pain, 84(1):95-103, January 2000.

[7] Antonius Rohlmann, Thomas Zander, Friedmar Graichen, Marcel Dreischarf, and Georg Bergmann. Measured loads on a vertebral body replacement during sitting. The spine journal : official journal of the North American Spine Society, 11(9):870-5, September 2011.

[8] Andrew Claus, Julie Hides, G Lorimer Moseley, and Paul Hodges. Sitting versus standing: does the intradiscal pressure cause disc degeneration or low back pain? Journal of electromyography and kinesiology : official journal of the International Society of Electrophysiological Kinesiology, 18(4):550-8, August 2008.

[9] Dieter Schmitter. Sitzen oder stehen? Ergonomische Gestaltung von Arbeitsplätzen. SUVA, 2 edition, March 2009.
[10] Barbara E Ainsworth et al. 2011 compendium of physical activities: a second update of codes and met values. Med Sci Sports Exerc, 43(8):1575-1581, Aug 2011.

[11] Allan Toomingas, Mikael Forsman, Svend Erik Mathiassen, Marina Heiden, and Tohr Nilsson. Variation between seated and standing/walking postures among male and female call centre operators. BMC public health, 12(1):154, January 2012.

[12] Dieter Breithecker. Arbeitsplatz Schule - Wie sieht das Klassenzimmer der Zukunft aus? Haltung und Bewegung, 25:17-22, 2005.

[13] Oliver Ludwig and Dieter Breithecker. Untersuchung zur Änderung der Oberkörper- durchblutung während des Sitzens auf Stühlen mit beweglicher Sitzfläche. Haltung und Bewegung, 3:5-12, 2008.

[14] Michael Haller, Christoph Richter, Peter Brandl, Sabine Gross, Gerold Schossleitner, Andreas Schrempf, Hideaki Nii, Maki Sugimoto, and Masahiko Inami. Finding the right way for interrupting people improving their sitting posture. In Proceedings of the 13th IFIP TC 13 international conference on Human-computer interaction - Volume Part II, INTERACT'11, pages 1-17, Berlin, Heidelberg, 2011. Springer-Verlag.

[15] Kathrin Probst, Jakob Leitner, Florian Perteneder, Michael Haller, Andreas Schrempf, and Josef Glöckl. Active office: Towards an activity-promoting office workplace design. In CHI '12: Proceedings of the 30th international conference extended abstracts on Human factors in computing systems, pages 21652170, New York, NY, USA, May 2012. ACM.

[16] P. A. Fenety, C. Putnam, and J. M. Walker. In-chair movement: validity, reliability and implications for measuring sitting discomfort. Appl Ergon, 31(4):383393, Aug 2000.

[17] Bilge Mutlu, Andreas Krause, Jodi Forlizzi, Carlos Guestrin, and Jessica Hodgins. Robust, low-cost, non-intrusive sensing and recognition of seated postures. Proceedings of the 20th annual ACM symposium on User interface software and technology, pages 149-158, 2007.

[18] Andreas Schrempf, Gerold Schossleitner, Thomas Minarik, Michael Haller, and Sabine Gross. PostureCare - Towards a novel system for posture monitoring and guidance. In Proceeding of the IFAC World Congress, volume 18, pages 593-598, 2010.

[19] Ying Zheng and J.B. Morrell. A vibrotactile feedback approach to posture guidance. IEEE Haptics Symposium, pages 351-358, 2010. 
[20] Donald D. Harrison, Sanghak O. Harrison, Arthur C. Croft, Deed E. Harrison, and Stephan J. Troyanovich. Sitting biomechanics part i: Review of the literature. Journal of Manipulative and Physiological Therapeutics, 22(9):594 - 609, 1999.

[21] Michael A. Adams and Patricia Dolan. Spine biomechanics. Journal of Biomechanics, 38(10):1972 1983, 2005.

[22] Thomas Minarik, Christopher Hofer, and Andreas Schrempf. A novel activity monitoring device for home rehabilitation applications. In Proceeding IASTED (764) Biomedical Engineering / 765: Telehealth /766: Assistive Technologies, 2012. 